\title{
Investigations of the Cellular Metabolism of Various Bacteria Utilizing Light-addressable Potentiometric Sensors (LAPS) Incorporated with Multi-chamber Structures
}

\author{
S. Dantism ${ }^{1,3}$, D. Röhlen ${ }^{1}$, T. Wagner ${ }^{1,2}$, P. Wagner ${ }^{3}$, M.J. Schöning ${ }^{1,2}$ \\ ${ }^{1}$ Institute of Nano- and Biotechnologies, Aachen University of Applied Sciences, Heinrich-Mußmann- \\ Straße 1, 52428 Jülich, Germany \\ 2 Institute of Complex Systems (ICS-8), Research Centre Jülich GmbH, 52425 Jülich, Germany \\ ${ }^{3}$ Department of Physics and Astronomy, Soft-Matter Physics and Biophysics Section, KU Leuven, \\ Celestijnenlaan 200 D, 3001 Leuven, Belgium \\ dantism@fh-aachen.de
}

\begin{abstract}
Light-addressable potentiometric sensors (LAPS) are field-effect-based potentiometric sensors, which are able to monitor analyte concentrations in a spatially resolved manner. Hence, a LAPS measuring system is a powerful tool to acquire a chemical image visualizing different concentrations of bio/chemical species in aqueous solutions such as for cellular metabolism studies. The planar sensor surface of LAPS allows a simple fixation of measurement chambers enabling simultaneous and multivariate analysis of different analyte solutions. In this work, 3D-printed photo-polymer-based (PPABS) multi-chamber structures were combined with LAPS to study the metabolic activity of Escherichia coli (E. coli) K12, Corynebacterium glutamicum and Lactobacillus brevis, as test microorganisms. In order to eliminate undesired influences (e.g., pH value variations of the medium and sensor signal drifts) during the measurements, differential measurements were performed to determine the acidification rates of those bacteria after glucose uptake. In order to improve the performance of the LAPS system, a novel FPGA-controlled illumination unit consisting of 16 smallsized tunable infrared laser-diode modules (LDMS) has been developed. Furthermore, a salt bridge chamber was built up and integrated with the LAPS measuring system providing multi-analyte measurements with a single $\mathrm{Ag} / \mathrm{AgCl}$ reference electrode.
\end{abstract}

Key words: light-addressable potentiometric sensor (LAPS), 3D-printed chambers, Escherichia coli K12, Corynebacterium glutamicum, Lactobacillus brevis, acidification rate, differential measurement, laser-diode modules, salt bridge chamber.

\begin{abstract}
Introduction
On-line monitoring of the metabolic activity of microorganisms can avoid costly process disturbances at an early stage in different biofermentation processes. For instance, process downtimes in biogas plants caused by metabolically inactive microorganisms can induce irreversible hindrances and costintensive interventions, which can be prevented by utilizing an efficient monitoring system. Short response time, small size, mass fabrication and solid-state nature of field-effect-based potentiometric sensors such as LAPS (lightaddressable potentiometric sensors) are representing advantages as promising candidates for those monitoring systems. In
\end{abstract}

general, LAPS with the appropriate recognition layer provides a spatially resolved detection of a certain analyte and can record 2D-chemical images of concentration changes of bio/chemical species on its flat surface. In this work, a LAPS-based multi-chamber measuring system with 16 laser-diode modules was developed, as shown in Fig. 1. The differential LAPS measurement principle was implemented to eliminate external influences such as temperature fluctuations, $\mathrm{pH}$ value variations of the stock solution and sensor signal drifts. By applying multi-chamber structures, simultaneous measurements were performed to determine the acidification rates of microorganisms such as Escherichia coli K12 
[1], Corynebacterium glutamicum and Lactobacillus brevis with regard to their cellular metabolization after glucose uptake. With the knowledge about the acidification behaviour of each microorganism in a multivariate analysis, a signal-response pattern can be realized. In this way, the sensor signal and the status of the biofermentation process can be correlated. Furthermore, a salt bridge chamber was built up and integrated with the LAPS allowing differential measurements with a single $\mathrm{Ag} / \mathrm{AgCl}$ reference electrode.

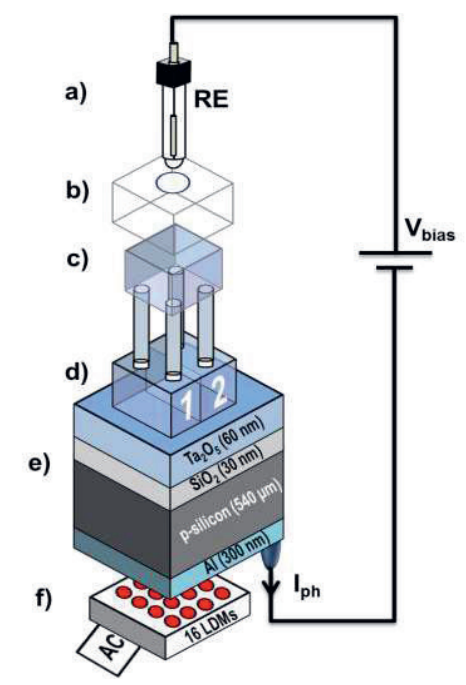

Fig. 1. Schematic of the multi-chamber differential measurement LAPS set-up. $\mathrm{Ag} / \mathrm{AgCl}$ reference electrode a), sealing cap b), salt bridge chamber $c$ ), two-chamber structure fixed on sensor surface d), sensor chip of $\mathrm{Al} / \mathrm{p}-\mathrm{Si} / \mathrm{SiO}_{2} / \mathrm{Ta}_{2} \mathrm{O}_{5} \mathrm{e}$ ), and light source based on 16 laser-diode modules f). 1: active sensor side with cells, 2: reference sensor side without cells. $I_{\text {ph }}$ : photocurrent, $V_{\text {bias: }}$ : bias voltage, AC: alternating current, LDMs: laser-diode modules, RE: reference electrode.

\section{Material and Methods}

Microorganism cultures were washed and resuspensioned in phosphate-buffered saline (PBS) solution (0.2 mM/pH, $\mathrm{pH} 7.4)$. Different glucose concentrations were prepared with the same buffer solution. A two-chamber LAPS measuring system was deployed for differential measurements, as assembled in Fig. 1. First, both chambers were loaded with $100 \mu \mathrm{l}$ of glucose solution $(0.17 \mathrm{mM}$ up to $1.67 \mathrm{mM})$. The salt bridge chamber was filled with $1 \mathrm{ml}$ of $3 \mathrm{M}$ $\mathrm{KCl}$ and was immersed into the two chambers passing the bias voltage through a $\mathrm{Ag} / \mathrm{AgCl}$ reference electrode to the sensor surface containing the glucose solution. After a conditioning phase of $10 \mathrm{~min}$ with the glucose concentration, $200 \mu$ l of cell suspensions (e.g., E. coli K12 with $4.8 \times 10^{9}$ cells in PBS solution) were pipetted into the first chamber (active sensor side with cells). In the second chamber (reference side without cells), $200 \mu$ l of the PBS solution were added. Eight laser-diode modules (LDMs) were considered for each sensor side. The differential signals were obtained by substracting the mean values of eight light spots applied for each sensor side. This measurement procedure was performed for all glucose concentrations (see also Fig. 2).

\section{Results and Discussion}

Fig. 2 exemplary represents five successive differential measurements with an ascending row of glucose concentrations and a constant cell number for $E$. coli K12 bacteria.

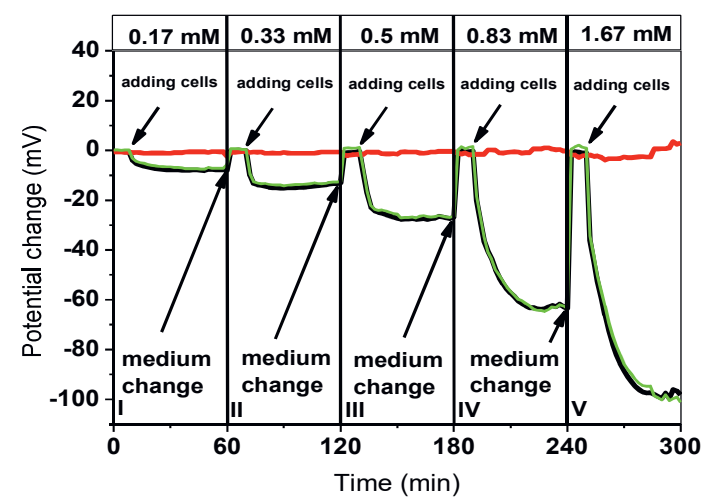

Fig. 2. Sensor signal for five successive differential LAPS measurements with ascending row of glucose concentrations. The cell number was constant $(4.8 \times$ $10^{9}$ cells) for each measurement. Black line: active sensor side with cells, red line: reference side without cells, green line: differential signal.

Increasing the glucose concentration increases the metabolic activity of $E$. coli $\mathrm{K} 12$ after glucose uptake. Thus, higher potential change rates $(\mathrm{mV} / \mathrm{min})$ and related acidification rates $(\Delta \mathrm{pH} / \mathrm{min})$ were observed by the LAPS. Furthermore, the metabolic activity of other bacteria, namely Corynebacterium glutamicum and Lactobacillus brevis have been studied utilizing the LAPS-based set-up (Fig.1). Results of all three types of bacteria will be compared and presented at the conference. The multichamber LAPS set-up enables multivariate analysis of the metabolic activity of bacteria on a single sensor chip.

\section{Acknowledgement}

The authors thank the German Federal Ministry of Food and Agriculture (project 22006613). We thank also the German Federal Ministry of Education and Research" (project 13N12585) for the partial financial support of this work.

\section{References}

[1] S. Dantism, S. Takenaga, T. Wagner, P. Wagner, M. J. Schöning, Differential imaging of the metabolism of bacteria and eukaryotic cells based on light-addressable potentiometric sensors, Electrochimica Acta 246, 234-241 (2017); doi: 10.1016/j.electacta.2017.05.196 